\title{
Non-strange baryons (CLAS)
}

\author{
Steffen Strauch ${ }^{1, *}$ for the CLAS Collaboration \\ ${ }^{1}$ University of South Carolina, Columbia, SC 29208
}

\begin{abstract}
Baryon spectroscopy is an essential tool in the study of nucleon resonances. The use of polarization observables can greatly clarify the spectrum of broad and overlapping nucleon excitations. The $N^{*}$ program with the CEBAF Large Acceptance Spectrometer (CLAS) at the Thomas Jefferson National Accelerator Facility includes experimental studies with linearly- and circularly-polarized tagged-photon beams, longitudinally- and transversely-polarized nucleon targets, and recoil polarizations. An overview of these experimental studies and recent results are presented.
\end{abstract}

\section{Introduction}

The CLAS Collaboration is pursuing an experimental study of baryon resonances with two components. The first is the spectroscopy of excited baryon states with photoproduction experiments. These experiments aim to search for new states and improve the understanding of known states. The experimental data are used in phenomenological analyses or theoretical models to extract resonance information. The extraction is a problem encompassing many short-lived, wide, and overlapping resonances with multiple decay-channels. It is particularly important to include in the analysis data from a variety of excitation and decay channels, as some of the missing states may couple only weakly to, e.g., the $\pi N$ final state. The CLAS experiments that are reported here include the $\pi^{0} p, \pi^{+} n, \eta p, \eta^{\prime} p, \omega p, K^{+} \Lambda, K^{*} \Lambda$, and $\pi^{+} \pi^{-} p$ reaction channels for reactions off the proton, and $\pi^{-} p, K^{0} \Lambda$, and $K^{0} \Sigma^{0}$ for quasi-free reactions off the neutron. The determination of a set of polarization observables, along with cross-section measurements, is essential in placing stringent constraints on the amplitude of the reaction. The second component of the CLAS $N^{*}$ program is the measurement of transition form factors of known resonances in electroproduction experiments to study their internal structure and confining potential. R. Gothe presented the CLAS and CLAS12 transition-form-factor experiments at this NSTAR 2019 conference.

The following gives a brief overview of recent examples of photoproduction experiments that were performed with the CEBAF Large Acceptance Spectrometer (CLAS) detector [1] at the Jefferson National Accelerator Facility (JLab). In these experiments, unpolarized, circularly, or linearly-polarized tagged [2] Bremsstrahlung photon beams were incident on unpolarized, longitudinally, or transversally polarized hydrogen or deuterium targets. The newly developed polarized targets were key to the success of the campaign. The frozen-spin target (FROST)

*e-mail: strauch@sc.edu
[3] provides dynamically polarized protons in TEMPOdoped butanol. In the g9a run, the average proton polarization was approximately $82 \%$ [4]. The dilution factor, the fraction of the number of free to the total number of protons that contribute to the reaction, depends on the reaction channel and kinematics; for the $\gamma p \rightarrow \pi^{+} n$ reaction, it was typically well above 0.5 . Spin-lattice relaxation times up to $4000 \mathrm{~h}$ were observed with FROST [3]. The polarized solid deuterium-hydride (HDice) $[5,6]$ target provided polarized neutrons. In the g14 run, the $D$ polarization of the HDice target averaged 25\% [7]. The spin relaxation time of the HDice target can reach years. Finalstate particles were detected in the CLAS or reconstructed through invariant and missing masses. The measured observables include total and differential cross-sections, as well as single- and double-polarization observables with combinations of a polarized beam, target, and (in the case of hyperon production) the polarization of the recoiling baryon.

\section{Photoproduction off the proton}

Measurements of the beam-target observable, $E$, in the $\gamma p \rightarrow \pi^{+} n$ reaction with circularly-polarized photons incident on longitudinally-polarized protons were the first results from the FROST experiment [4]. Over 900 data points were obtained in a center-of-mass energy range from $W=1.25 \mathrm{GeV}$ to $2.23 \mathrm{GeV}$. For most of the covered energy range, previous partial-wave analyses (PWA) showed significant deviations; only at low energies, the agreement with the data was fair. The inclusion of the data in new analyses led to a better description of the data and an improved amplitude determination. These more tightly constrained amplitudes also help fix the $\pi N$ components of broader multi-channel analyses. One particularly interesting result is the strengthened the evidence for the, at the time, poorly known $\Delta(2200) 7 / 2^{-}$resonance by improving the Bonn-Gatchina (BnGa) fit at the highest energies [8]. The mass of the $\Delta(2200) 7 / 2^{-}$resonance is significantly 
higher than the mass of its parity partner $\Delta(1950) 7 / 2^{+}$, which is the lowest-mass $\Delta^{*}$ resonance with spin-parity $J^{P}=7 / 2^{+}$. The observation conflicts with the prediction of models assuming a phase transition in high-mass resonances. The $\Delta(1950) 7 / 2^{+}$is now listed as a three-star resonance by the Particle Data Group [9].

Two CLAS analyses of single-pion photoproduction data from FROST have recently been completed and are being prepared for publication. The first is the extraction of the beam, $T$, and beam-target, $F$, polarization observables in $\gamma p \rightarrow \pi^{0} p$ from data with circularly-polarized photons and transversally-polarized protons [10]. The results are in good agreement with previous experimental data but extend to much higher energies, up to about $W=2.5 \mathrm{GeV}$. Although these results have not yet been included in fits of recent partial-wave analyses, the PWA solutions are already in fair agreement with the data, even at the highest covered energies. The second analysis determined the beam-target observable, $G$, from data with linearly polarized photons on a longitudinally polarized proton target in the $\pi^{0} p$ and $\pi^{+} n$ channels for energies from $W=1.425 \mathrm{GeV}$ to $2.290 \mathrm{GeV}$ [11]. While present PWA predictions are in fair agreement with the data, they do not describe the $G$ data well at higher energies. Figure. 1 shows example angular distributions for $G$ in the $\pi^{+} n$ channel for two out of the 23 energy bins in the experiment.
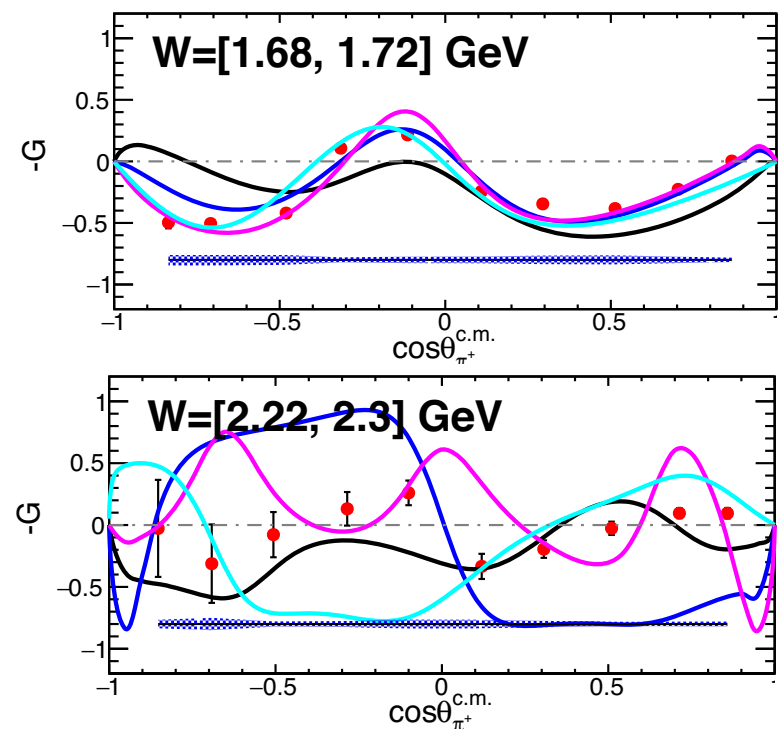

Figure 1. Beam-target polarization observable $-G$ in the $\gamma p \rightarrow$ $\pi^{+} n$ reaction from FROST for two selected energy bins out of 23 (red data points) [11]. The curves are predictions from SAID MA19 (blue) [12], MAID (magenta), GW-Jülich (cyan) [13], and $\mathrm{BnGa}$ (black) $[14,15]$.

The $\eta$ meson is an isoscalar particle $(I=0)$, and its production in the $s$-channel process can proceed via $N^{*}$ states with $I=\frac{1}{2}$ but not via $\Delta^{*}$ resonances with $I=\frac{3}{2}$; the channel $\eta p$ serves as an isospin filter. The polarization observable $E$ has been measured with FROST in the $\gamma p \rightarrow$ $\eta p$ reaction from threshold to $W=2.15 \mathrm{GeV}$ [16]. Initial investigation of these results with the Jülich-Bonn dynamical coupled-channel model show pronounced changes in the description of this observable when these new CLAS data are included. This analysis gave a good description of the data without the need for an additional and previously suggested narrow resonance near $1.68 \mathrm{GeV}$. The CLAS Collaboration has also measured the linearlypolarized photon-beam asymmetry, $\Sigma$, in the $\gamma p \rightarrow \eta p$ and $\gamma p \rightarrow \eta^{\prime} p$ reactions. The data significantly extend the range of previous measurements to energies up to about $W=2.08 \mathrm{GeV}$ [17]. Based on the Jülich-Bonn approach and the BnGa partial-wave formalism, initial analyses of the data corroborate the evidence for the nucleon resonances $N(1895) 1 / 2^{-}, N(1900) 3 / 2^{+}, N(2100) 1 / 2^{+}$, and $N(2120) 3 / 2^{-}$[17]. The analysis by Anisovich et al. [18] indicates that those four resonances are the most significant contributors to the $\eta^{\prime}$ photoproduction process. This analysis of the CLAS data also allowed for the determination of the decay branching ratios of these resonances into $N \eta^{\prime}$ final states. The Particle Data Group now recognizes these states as three- and four-star resonances [9].

Like the $\eta$ and $\eta^{\prime}, \omega$ mesons have isospin zero. The $\omega$ photoproduction selects isospin- $\frac{1}{2}$ resonances in the nucleon resonance spectrum. The CLAS Collaboration has studied cross-sections and numerous polarization observables in the $\gamma p \rightarrow \omega p$ reaction using the $\omega \rightarrow \pi^{+} \pi^{-} \pi^{0}$ decay mode. In a measurement with circularly polarized photons and the longitudinally polarized FROST target, the helicity asymmetry $E$ was determined from threshold to about $W=2.3 \mathrm{GeV}$ [19]. An analysis of the data within the $\mathrm{BnGa}$ framework found as leading partial waves at the reaction threshold the $3 / 2^{+}$, identified with the sub-threshold $N(1720) 3 / 2^{+}$resonance, and $5 / 2^{+}$ partial waves. Pomeron $t$-channel exchange was seen to grow with energy and to dominate all other contributions above $W \approx 2 \mathrm{GeV}$; $t$-channel pion exchange was found to remain small in the studied energy range [19]. The magnitudes of the interference terms between the leading Pomeron-exchange and the resonant portion of the $J^{P}=3 / 2^{+}$partial waves, as well as the smaller waves with $J^{P}=1 / 2^{-}, 3 / 2^{-}$, and $5 / 2^{+}$, were fixed with the help of recent CLAS measurements of the photon-beam asymmetry $\Sigma$ [20]. The data significantly increase the size of the database for this observable and extend its coverage to energies up to $W=2.1 \mathrm{GeV}$. First-time measurements of the target asymmetry $T$ in the $\gamma p \rightarrow \omega p$ reaction were obtained with the FROST target from the reaction threshold up to $2.8 \mathrm{GeV}$ [21]. The observed asymmetries are rich in structures and significant; with $|T|$ reaching $0.3-$ 0.4 in the third-resonance region. The FROST data also allowed to determine a new set of results for the observable $\Sigma$ [21] that were found to be in overall fair agreement with previous data, including the earlier CLAS data [20]. Finally, the double-polarization observables $F, H$, and $P$ in $\omega$ photoproduction have been obtained from the FROST experiment using circularly polarized and linearly polarized photons [22]. The results of partial-wave analyses, including these new data, provide evidence for several poorly known states; the recently added $N(1875) 3 / 2^{-}$ and $N(2120) 3 / 2^{-}$states, the new $N(1880) 1 / 2^{+}$, and the poorly established $N(2000) 5 / 2^{+}$. The latter state was also 
observed in a single-channel partial-wave analysis by the CLAS Collaboration [23].

Hyperon photoproduction is an important tool in the study of nucleon resonances. The parity-violating weak decay of hyperons allows access to recoil polarization observables through the measurement of the decay-proton angular distribution. Earlier CLAS measurements of the hyperon-photoproduction reactions $\gamma p \rightarrow K^{+} \Lambda$ and $\gamma p \rightarrow$ $K^{+} \Sigma^{0}$ included the determination of the $\Lambda$ recoil polarization $P$ from measurements with unpolarized photon beams $[24,25]$, as well as the polarization-transfer observables $C_{x}$ and $C_{z}$ with circularly-polarized beams [26]. Among other data, these CLAS findings were particularly important in an analysis by the BnGa group [27, 28] that established further evidence for the, at the time, poorly known $N(1900) 3 / 2^{+}$resonance. This resonance is predicted by symmetric three-quark models but is not expected to exist in some quark-diquark models [28]. By now, this resonance is recorded as a firmly established four-star resonance by the Particle Data Group [9]. New hyperonphotoproduction data for the reactions $\gamma p \rightarrow K^{+} \Lambda$ and $\gamma p \rightarrow K^{+} \Sigma^{0}$ from CLAS were extracted from measurements with linearly-polarized photons off an unpolarized proton target with energies up to $W \approx 2.2 \mathrm{GeV}$ [29]. The linearly-polarized beam, together with the recoil polarization of the hyperon, give access to five polarization observables: $\Sigma, P, T, O_{x}$, and $O_{z}$. These data greatly increase the world database in accuracy and kinematic coverage; the beam-recoil observables, $O_{x}$ and $O_{z}$, in the $K^{+} \Sigma^{0}$ channel have not been reported before. An earlier 2014 solution of the $\mathrm{BnGa}$ partial-wave analysis did not describe most data from this experiment. An updated solution that included the new CLAS data and additional $N^{*}\left(3 / 2^{+}\right)$and $N^{*}\left(5 / 2^{+}\right)$resonances describes the $K \Lambda$ data well, whereas the agreement with the $K \Sigma$ data is fair [29]. Hyperon photoproduction data from the FROST experiment have been analyzed [30]; final results are not yet available.

Furthermore, the CLAS Collaboration reported first measurements of the spin-density matrix elements of $K^{*+}(892)$ mesons in the reaction $\gamma p \rightarrow K^{*+} \Lambda$ where the $K^{*+}$ was identified in the observation of $K^{*+} \rightarrow K_{S} \pi^{+}$with a subsequent $K_{S} \rightarrow \pi^{+} \pi^{-}$[31]. The measurement covered a photon-energy range from threshold to $3.9 \mathrm{GeV}$. The results from this experiment - differential cross-sections, spin density matrix elements, and the $\Lambda$ recoil polarization - were analyzed in the BnGa framework. This analysis found very significant contributions of the nucleon resonances $N(1895) 1 / 2^{-}$and $N(2100) 1 / 2^{+}$to the reaction, and some indications for three new resonances that decay into $K^{*} \Lambda$ [31].

Highly excited nucleons, in the mass region above $1.6 \mathrm{GeV}$, have substantial branching ratios into the $\pi \pi N$ final state; the decay proceeds typically through either the $\pi \Delta$ or $\rho N$ intermediate states. The study of $\pi \pi N$ photoproduction is, therefore, a sensitive tool in the search for new baryon states that couple weakly to other decay channels, especially to $\pi N$. The CLAS Collaboration recently reported on the first measurement of nine single-differential cross sections for the $\gamma p \rightarrow \pi^{+} \pi^{-} p$ reaction in the energy range from $W=1.6 \mathrm{GeV}$ to $2.0 \mathrm{GeV}$ [32]. The various cross-sections are differential in the invariant masses, as well as polar and azimuthal angles for different pairs of final-state particles. The extensive data set was analyzed with the updated Jefferson Lab-Moscow State University meson-baryon reaction model, and a good description of the data was achieved [32]. The analysis provided the first extraction of the photocouplings of all prominent nucleon resonances in this mass region from $\pi^{+} \pi^{-} p$ photoproduction data. Compared to previous $\pi N$ analyses, the accuracy of the photocouplings of the resonances with dominant decay into the $\pi \pi N$ final state, $\Delta(1620) 1 / 2^{-}, \Delta(1700) 3 / 2^{-}$, $N(1720) 3 / 2^{+}$, and $\Delta(1905) 5 / 2^{+}$, were greatly improved [32].

Beyond differential cross sections, polarization observables provide further constraints on the extraction of the amplitudes of the photoproduction process. A review of the polarization observables for the two-pion production off the nucleon is given in Ref. [33]. The CLAS Collaboration was first to study the $\pi^{+} \pi^{-} p$ photoproduction with a circularly polarized beam and to measure the beam-helicity asymmetry, $I^{\odot}$, in the energy range between $W=1.35$ and $2.30 \mathrm{GeV}$ [34]. The use of linearly polarized photons and the FROST target allows for the extraction of a large number of polarization observables. The FROST group is working on the determination of twelve polarization observables determined from $\gamma p \rightarrow p \pi^{+} \pi^{-}$data; preliminary results are reported in Refs. [35, 36]. Figure 2 illustrates the quality of data that is expected from those analyses. Here, the azimuthal angular distributions of the double-polarization observable $P_{y}^{\odot}$ are shown for various polar angular ranges for one center-of-mass energy bin raging from $W=1550 \mathrm{MeV}$ to $1650 \mathrm{MeV}$ [36]. The data are compared with model calculations by Fix [37] and are fitted with a low-order polynomial in $\sin \Phi^{*}$. This particular observable is accessible in measurements with circularly polarized photons off transversally polarized protons. Other observables accessible with these experimental conditions include $I^{\odot}, P_{x}^{\odot}, P_{x}$ and $P_{y}$. [33].

\section{Photoproduction off the neutron}

Compared to the scale of photoproduction data with proton targets, the world database for neutron targets is sparse. However, data from both proton and neutron targets are needed, for example, to completely specify the amplitude of the $\gamma N \rightarrow \pi N$ reaction. The amplitude of the reaction contains two parts with isospin $I=\frac{1}{2}$ and $I=\frac{3}{2}$, respectively. While the excitation of $I=\frac{3}{2} \Delta^{*}$ states can be entirely determined from proton target data, measurements with both neutron and proton targets are required to deduce the isospin $I=\frac{1}{2}$ amplitudes, and separate $\gamma p N^{*}$ and $\gamma n N^{*}$ couplings [38]. The couplings provide essential guides to nucleon-structure models. A complication associated with the extraction of neutron data from experiments on nuclear targets, like the deuteron, is the model-dependent interpretation of the results.

The CLAS Collaboration measured the differential cross sections for the quasi-free $\gamma d \rightarrow \pi^{-} p(p)$ reaction (with proton spectator) in the energy range from $W=$ $1.31 \mathrm{GeV}$ to $2.37 \mathrm{GeV}$. After corrections of the $\gamma d$ data 

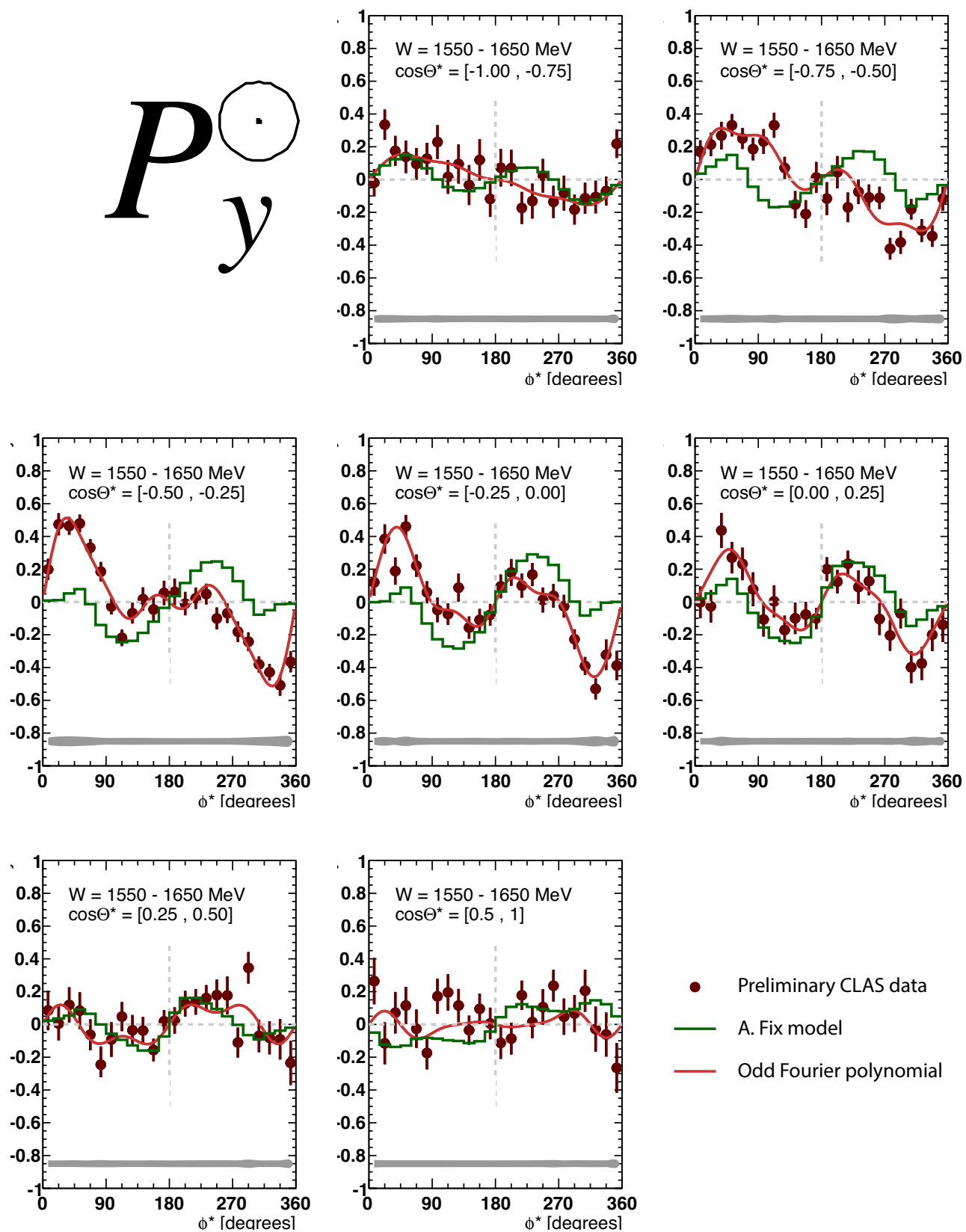

- Preliminary CLAS data

- A. Fix model

Odd Fourier polynomial

Figure 2. Preliminary $\Phi^{*}$ azimuthal angular distributions of the beam-target polarization observable $P_{y}^{\odot}$ for the $\gamma p \rightarrow \pi^{+} \pi^{-} p$ reaction in the energy range $W=1550 \mathrm{MeV}$ to $1650 \mathrm{MeV}$ [36]. The seven panels correspond to bins in the polar angle $\Theta^{*}$. The solid green histograms are CLAS-acceptance averaged results of model calculations from A. Fix [37]. To guide the eye, the data are also fitted with low-order polynomials in $\sin \Phi^{*}$ (red curves). The angles $\Phi^{*}$ and $\Theta^{*}$ are, respectively, the azimuthal and polar angles of the final-state $\pi^{+}$; see Ref. [36] for details.

for final-state interactions, 8428 differential cross-section data points were extracted for the $\gamma n \rightarrow \pi^{-} p$ reaction, nearly tripling the corresponding world statistics [12]. A SAID multipole analysis was performed on the data set and resulted in the extraction of several photo-decay amplitudes $N^{*} \rightarrow \gamma n$ with small uncertainties, and, in particular, it allowed the first-ever determination of the excited neutron multipoles for the $N(1440) 1 / 2^{+}, N(1535) 1 / 2^{-}$, $N 1650) 1 / 2^{-}$, and $N(1720) 3 / 2^{+}$resonances [12]. CLAS data with a linearly polarized photon beam have been used to extract about 1200 data points of the polarization ob- servable $\Sigma$ in $\gamma d \rightarrow \pi^{-} p(p)$ covering an energy range from $W=1.62 \mathrm{GeV}$ to $2.36 \mathrm{MeV}$ [39]. The final results are not yet available.

The CLAS Collaboration has taken production data with circularly- and linearly-polarized photons off a polarized solid deuterium-hydride target (HDice) [5, 6] up to center-of-mass energies of $W \approx 2.3 \mathrm{GeV}$. The run conditions were optimized for polarized neutron reactions. A first result is the determination of the beam-target doublepolarization asymmetry $E$ in the $\gamma d \rightarrow \pi^{-} p(p)$ reaction [7]. New partial-wave analyses that included the data re- 
sulted in updated $\gamma n N^{*}$ photocouplings [7]. Additional work on the experimental data is ongoing to extract the polarization observables $\Sigma$ and $G$. Preliminary results for $\Sigma$ are consistent with existing partial-wave analyses, whereas the results for $G$ deviate strongly [40]. The data hint at the prospect of providing valuable constraints for new partialwave analyses.

Hyperon photoproduction data off the proton were key to elucidate the nucleon resonance spectrum. Now, the CLAS Collaboration has reported on the first measurement of the differential and total cross sections for the $\gamma d \rightarrow K^{0} \Lambda(p)$ reaction with energies up to $W=2.5 \mathrm{GeV}$ [41]. Consistent cross-sections were extracted from two different CLAS experiments with a partial overlap in the photon-energy range. First, partial-wave analyses were performed that describe the data without the introduction of new resonances. Two independent solutions from the BnGa-fit describe the cross-section data equally well [41]. The induced polarization $P$ and the polarization-transfer coefficients $C_{x}$ and $C_{z}$ were also extracted for the $K^{0} \Lambda(p)$ channel from data with circularly polarized photons on a deuterium target in the energy range up to $W=2.4 \mathrm{GeV}$ [42]. The preliminary results in Fig. 3 indicate that $C_{z} \approx 1$ at forward kaon-angles for photon energies up to $1.4 \mathrm{GeV}$. The preliminary data have been compared to results from the ANL Osaka and BnGa groups, and the significant deviations hint at the potential of these data to further constrain the $N^{*}$ amplitude analysis. The beam-target helicity asymmetry $E$ in $K^{0} \Lambda$ and $K^{0} \Sigma^{0}$ photoproduction on the neutron were extracted for the first time [43]. The experiment made use of the HDice target and the identification of $\pi^{+} \pi^{-} \pi^{-} p(X)$ with intermediate hyperons in the final state in the CLAS. The data cover an energy range from $W=1.70 \mathrm{GeV}$ to $2.34 \mathrm{GeV}$. Regrettably, this analysis is limited by small statistics.

\section{Summary}

Much of the progress in the understanding of the baryon spectrum is owed to a large amount of new photoproduction data. The data include reaction cross sections and polarization observables from experiments with polarized beams or targets, as well as recoil polarization observables. Many final states have been studied in the experiments. The progress in the field is evident in the increased number of known $N$ and $\Delta$ resonances, as reported by the Particle Data Group [9]. The $N^{*}$ program of CLAS has been a critical contributor to these studies, and ongoing, and future analyzes of CLAS data will continue to elucidate the nucleon resonance spectrum.

\section{References}

[1] B.A. Mecking et al., Nucl. Instrum. Meth. A503, 513 (2003)

[2] D. Sober et al., Nucl. Instrum. Meth. A 440, 263 (2000)

[3] C. Keith, J. Brock, C. Carlin, S. Comer, D. Kashy, J. McAndrew, D. Meekins, E. Pasyuk, J. Pierce, M. Seely, Nucl. Instrum. Meth. A 684, 27 (2012)
[4] S. Strauch et al. (CLAS), Phys. Lett. B750, 53 (2015), 1503.05163

[5] C.D. Bass et al., Nucl. Instrum. Meth. A737, 107 (2014)

[6] M.M. Lowry et al., Nucl. Instrum. Meth. A815, 31 (2016), 1901.04548

[7] D. Ho et al. (CLAS), Phys. Rev. Lett. 118, 242002 (2017), 1705.04713

[8] A.V. Anisovich, V. Burkert, J. Hartmann, E. Klempt, V.A. Nikonov, E. Pasyuk, A.V. Sarantsev, S. Strauch, U. Thoma, Phys. Lett. B766, 357 (2017), 1503.05774

[9] M. Tanabashi et al. (Particle Data Group), Phys. Rev. D98, 030001 (2018)

[10] H. Jiang, S. Strauch (CLAS), Few Body Syst. 59, 125 (2018)

[11] N. Zachariou, D. Watts, L. Zana, J. McAndrew, CLAS Analysis

[12] P.T. Mattione et al. (CLAS), Phys. Rev. C96, 035204 (2017), 1706.01963

[13] D. Rönchen, M. Döring, U.G. Meißner, Eur. Phys. J. A54, 110 (2018), 1801. 10458

[14] V. Sokhoyan et al. (CBELSA/TAPS), Eur. Phys. J. A51, 95 (2015), [Erratum: Eur. Phys. J.A51,no.12,187(2015)], 1507.02488

[15] A.V. Anisovich et al., Eur. Phys. J. A52, 284 (2016), 1604.05704

[16] I. Senderovich et al. (CLAS), Phys. Lett. B755, 64 (2016), 1507.00325

[17] P. Collins et al. (CLAS), Phys. Lett. B771, 213 (2017), 1703.00433

[18] A.V. Anisovich, V. Burkert, P.M. Collins, M. Dugger, E. Klempt, V.A. Nikonov, B.G. Ritchie, A.V. Sarantsev, U. Thoma, Phys. Lett. B772, 247 (2017), 1706.05144

[19] Z. Akbar et al. (CLAS), Phys. Rev. C96, 065209 (2017), 1708.02608

[20] P. Collins et al. (CLAS), Phys. Lett. B773, 112 (2017), 1706.04280

[21] P. Roy et al. (CLAS), Phys. Rev. C97, 055202 (2018), 1711.05176

[22] P. Roy et al. (CLAS), Phys. Rev. Lett. 122, 162301 (2019), 1812.02106

[23] M. Williams et al. (CLAS), Phys. Rev. C80, 065209 (2009), 0908. 2911

[24] J.W.C. McNabb et al. (CLAS), Phys. Rev. C69, 042201 (2004), nucl-ex/0305028

[25] M.E. McCracken et al. (CLAS), Phys. Rev. C81, 025201 (2010), 0912. 4274

[26] R.K. Bradford et al. (CLAS), Phys. Rev. C75, 035205 (2007), nucl-ex/0611034

[27] A.V. Sarantsev, V.A. Nikonov, A.V. Anisovich, E. Klempt, U. Thoma, Eur. Phys. J. A25, 441 (2005), hep-ex/0506011

[28] V.A. Nikonov, A.V. Anisovich, E. Klempt, A.V. Sarantsev, U. Thoma, Phys. Lett. B662, 245 (2008), 0707.3600 


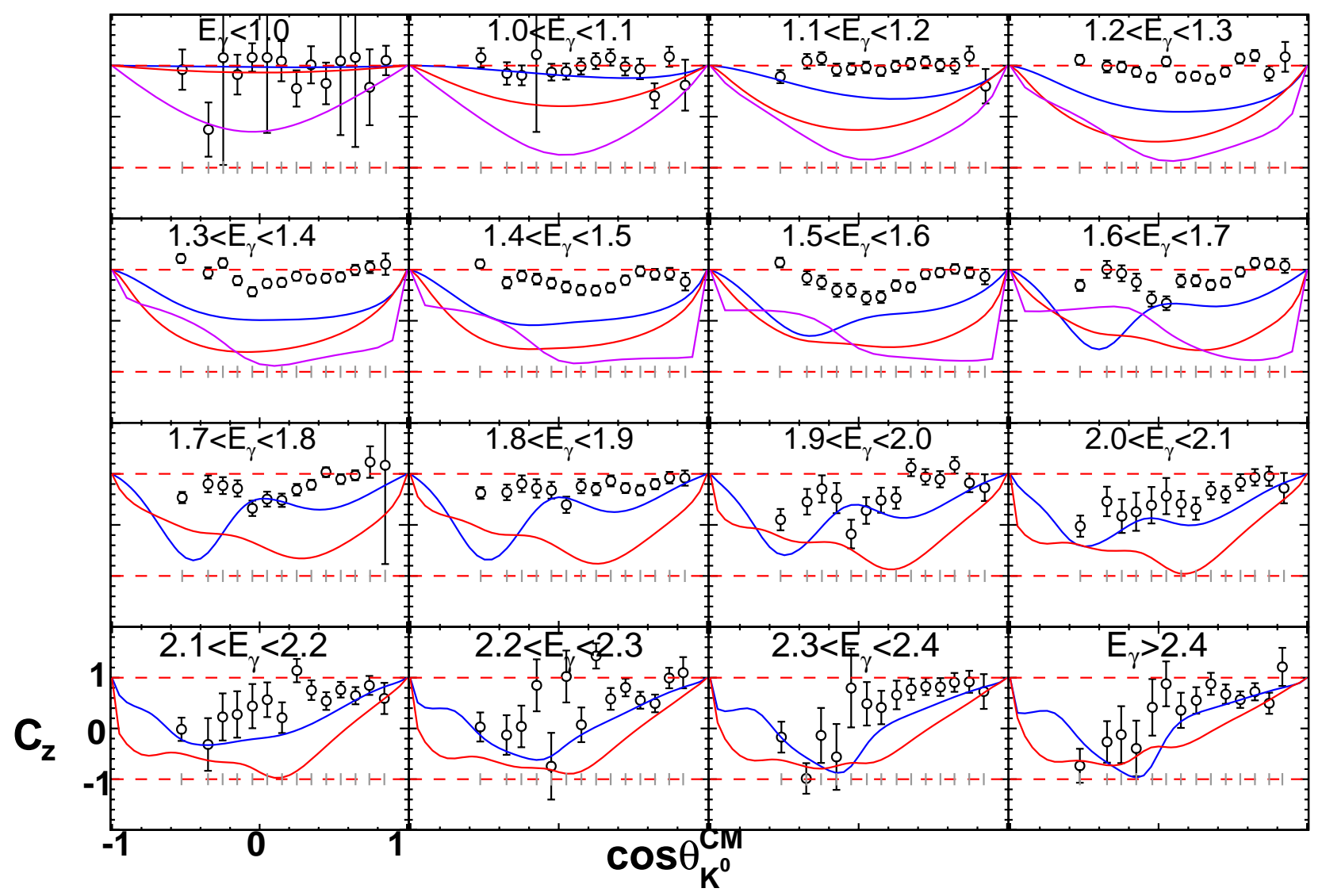

Figure 3. Preliminary $K^{0}$-angular distributions of the polarization-transfer observable $C_{z}$ in the $\gamma d \rightarrow K^{0} \Lambda(p)$ reaction (open circles) $[42,44]$. The panels show results from various photon-energy bins where the maximum energy is about $2.6 \mathrm{GeV}$. The preliminary data are compared with analysis solutions. The blue and the red curves show results from the BnGa group; the pink curve is from ANL Osaka [45].

[29] C.A. Paterson et al. (CLAS), Phys. Rev. C93, 065201 (2016), 1603.06492

[30] N.K. Walford, JPS Conf. Proc. 17, 062005 (2017)

[31] A.V. Anisovich et al. (CLAS), Phys. Lett. B771, 142 (2017)

[32] E. Golovatch et al. (CLAS), Phys. Lett. B788, 371 (2019), 1806.01767

[33] W. Roberts, T. Oed, Phys.Rev. C71, 055201 (2005), nucl-th/0410012

[34] S. Strauch et al. (CLAS Collaboration), Phys. Rev. Lett. 95, 162003 (2005), hep-ex/0508002

[35] S. Park (CLAS), AIP Conf. Proc. 1432, 401 (2012)

[36] L.A. Net, S. Strauch (CLAS), Few Body Syst. 59, $123(2018)$
[37] A. Fix, H. Arenhovel, Eur. Phys. J. A25, 115 (2005), nucl-th/0503042

[38] A.M. Sandorfi, Few Body Syst. 60, 10 (2019)

[39] D. Sokhan, CLAS Analysis

[40] H.Y. Lu (CLAS, g14 Analysis Group), Few Body Syst. 60, 18 (2019)

[41] N. Compton et al. (CLAS), Phys. Rev. C96, 065201 (2017), 1706.04748

[42] C. Gleason, Y. Ilieva (CLAS), Few Body Syst. 59, 129 (2018)

[43] D.H. Ho et al. (CLAS), Phys. Rev. C98, 045205 (2018), 1805.04561

[44] C. Gleason, private communication (2019)

[45] H. Kamano, S.X. Nakamura, T.S.H. Lee, T. Sato, Phys. Rev. C88, 035209 (2013), 1305. 4351 